\title{
MONTEIRO LOBATO \& ISAAC GOLDBERG : A AMÉRICA LATINA NA AMÉRICA DO NORTE ${ }^{1,2}$
}

\author{
Marisa Lajolo \\ marisal@uol.com.br
}

Entre junho de 1927 e dezenove de março de 1931, Monteiro Lobato (1882-1948) viveu em Nova Iorque. Recém saído da falência da

1. Versão anterior deste texto foi apresentada no Special Brazil Seminar, junto ao Harvard Brazil Studies Program (David Rockfeller Center for Latin American Studies) em novembro de 2010. A autora expressa seus agradecimentos ao Prof. Aaron Litvin, ao Prof. Nicolau Sevcenko e à Prof. Clemence M.Joüet-Pastré pelo convite e à Profa. Naomi Moniz pelo instigante debate posterior à apresentação.

2. $\mathrm{O}$ desenvolvimento da pesquisa da qual resultou este ensaio contou com apoio do CNPq (Bolsa Produtividade em Pesquisa, proc. 350 140 / 1994) e da FAPESP (Projeto Temático "Monteiro Lobato (1882-1948) e outros modernismos brasileiros "proc. 02/o8819-4) . Alguns dados aqui mencionados foram levantados em estágio de pesquisa desenvolvido junto ao Dartmouth College. A autora registra seus agradecimentos aos professores doutores Rodolfo Franconi e Raul Bueno Chavez pelo convite e pela hospitalidade, e ao Dr. Miguel Valladares, pelo inestimável auxílio para a pesquisa em bibliotecas e bancos de dados. Para outros resultados parciais da mesma pesquisa consultar Lajolo, M. A Brazilian writer [Monteiro Lobato] in New York apud Brazil/Brasil (o103-751X) volume 34,série 19,2006. p.17-31 ; Lajolo, M. Um brasileiro em trânsito apud Ilha do Desterro (UFSC) 2009 p. 37-57. ISSN 0101- 4846. 
Companhia Gráphico-Editora Monteiro Lobato ${ }^{3}$ e tendo já fundado (em sociedade com Octales Marcondes) a Companhia Editora Nacional havia sido nomeado, pelo presidente Washington Luis, Adido Comercial do Consulado Brasileiro.

A nomeação explica sua longa permanência nos Estados Unidos com a família (esposa, quatro filhos e uma empregada), permanência da qual dá conta em seu livro América, publicado em 1932 e que teve circulação bastante representativa ${ }^{4}$ mesmo para padrões atuais do mercado brasileiro:

\begin{tabular}{|c|c|}
\hline ANO & TIRAGEM \\
\hline 1932 & 6.000 \\
\hline 1934 & 3.000 \\
\hline 1937 & 2.000 \\
\hline
\end{tabular}

De qual Estados Unidos fala Monteiro Lobato aos mais de dez mil brasileiros que leram o livro na terceira década do século XX ?

Monteiro Lobato fala de uma América dourada e sedutora.

Assim como para narrar o velório do pai, o falso memorialista Brás Cubas informa seus leitores que Isto que parece um simples inventário, eram notas que eu havia tomado para um capítulo triste e vulgar que não escrevo ${ }^{5}$, para dar conta dos Estados Unidos comentados por Lobato segue abaixo um inventário (alfabeticamente disposto) de nomes próprios presentes no livro:

Alimony Club, Biblioteca do Congresso, Biblioteca Pública, Boston, British Imperial War Museum, Broadway Temple, Brooklyn, Cafeteria Califórnia, Capitólio, Capitólio (Albany), Casa das pontas, Catedral do Livro, Cemitério de Arlington, Chicago), Child's (lanchonete), Chrysler Building, túneis do metrô novaiorquino, Cinema Rialto, City Hall, Columbia, Coney Island, Cornel, Detroit Golf Club , Detroiter, East Hall, Edifício York Herald, Empire State Building, Equitable Building, Evening graphic, Filadélfia, Ford Motor

3. Para o estudo do processo de falência da Companhia Graphico Editora Monteiro Lobato, consultar Bignotto, C.C. Novas perspectivas sobre as práticas editoriais de Monteiro Lobato (1918-1925). Tese de Doutorado. Universidade Estadual de Campinas. Instituto de Estudos da Linguagem. 2007. Disponível em http://cutter.unicamp.br/ document/?code=vtlsooo410260

4. Dados extraídos de correspondência da Cia Editora Nacional para Monteiro Lobato. Cf. (Cedae MLb 3.2.00407cx8)

5. Machado de Assis. Obra completa . 3 vol. RJ Ed. José Aguilar Ltda. 1962 . vol I p. 560. 
Company, Fordhan, Fresh Air Taxi Cab, Gerry House, Gettsburg, Grand Central, Greenwich Village, Harlem, Harvard, Holland Tunnell, Hot Springs (Arkansas), Hudson, Igreja Congregacional de Manhattam, Igreja-Restaurante, Instituto Tecnológico de Massachussets, Lincoln Memorial, Macy's / Gimbels / Altman, Maryland, Virginia, Pensylvania, Kansas, Idaho e Ohio, Minesotta, Montanhas de Virgínia, Museu Comercial, Museu da Cruz Vermelha (em New York), Newark (subúrbio de NY), New England, Riverside Drive, Pallissade Park, Potomac, Princenton, Wilmington, Peekskill, Pensylvania Station, Pirate's Den, Ponte Monumental, Quinta Avenida, Riverside Drive, Rua 72, Roosevelt Field, Roxy, Rua Christofer, Rua Greenwich, Rua Hudson (em Long Beach), Ruskin, Sala de Leitura, São Francisco , Scardale, Sede General Motors, South Hall, Stanford, Stock Exchange de New York, Syracuse, Tarrytown (margem do rio Hudson), Texas, Little Rock (Arkansas), Trinity Church, Union Station, Universidade de Wisconsin, Walden Pond, Woolworth, Yale ${ }^{6}$

Cidades, monumentos, edifícios, instituições, bairros, linhas e estações de metrô, ruas e rios são o cenário a partir do qual Monteiro Lobato comenta a América (que vê ao vivo), ao lado do Brasil que, da América, vê a distância.

E que Brasil seria este?

Como funcionário do consulado brasileiro, Monteiro Lobato estava bastante exposto às representações do Brasil construídas pelo discurso oficial. Mas parece ignorá-las, quando não desconstruí-las.

Em agosto de 1927, carta de Octavio Mangabeira - então Ministro das Relações Exteriores - ao (então) Embaixador do Brasil na América do Norte, Gurgel do Amaral é sugestiva de um Brasil latino-americano que queria fortalecer-se, ainda que simbolicamente:

Senhor Embaixador, Tenho a honra de remeter a Vossa Excelência a inclusa copia de uma informação da Secção dos Negócios Políticos e Diplomáticos da América sobre o uso da língua portuguesa na União das Republicas Americanas. 2. Rogo a Vossa Excelência a bondade de examinar esse assunto, sobre o qual estimaria conhecer o seu parecer. Aproveito este ensejo para reiterar a Vossa Excelência os protestos da minha alta estima e mais distinta consideração. Octavio Mangabeira ${ }^{7}$

6. Enumeração organizada a partir e levantamento de Liane Schiavon , com Bolsa Trabalho da Unicamp 2002-2005 - realizado com vistas à organização da homepage disponível em http://www.unicamp.br/iel/monteirolobato

7. MUSEU DO ITAMARATY. SECRETARIA DE ESTADO DAS RELAÇÕES EXTERIORES. Em 19 de Agosto de 1927 NO. PX/ 17 Índice: $\mathrm{O}$ uso da língua portuguesa na União das Republicas Americanas. Todos os itálicos das citações dos documentos transcritos são meus, exceto indicado de outra forma. 
Três meses depois, em novembro do mesmo ano, nova carta sugere parceria e troca de informações políticas entre Brasil e Estados Unidos: o ministério informa a embaixada de sentimentos latino-americanos em curso na América Latina:

\begin{abstract}
Senhor Embaixador, Pelo Despacho confidencial PA/13 de julho último, dei conhecimento a Vossa Excelência da noticia aqui recebida da nossa Embaixada em Santiago, segundo a qual o México capitaneava, á frente dos países de América Central, um movimento contra os Estados Unidos da América na próxima Conferência Internacional Americana. Essa noticia teve-a o Embaixador Roças do Sub-Secretario das Relações Exteriores do Chile.

Acusando o recebimento daquele Despacho, disse-me Vossa Excelência, em substancia, no seu Officio Confidencial número 274, de 28 de agosto, que nada via aí pudesse corroborar os vaticínios pessimistas correntes em Santiago; e que os Estados Unidos da América, embora merecedores da oposição que lhes moviam certas correntes centro-americanas, acreditavam, contudo, que o prestígio de que goza seu Governo, e a sensatez das delegações $(. . .)^{8}$
\end{abstract}

Dentre iniciativas diplomáticas - como as sugeridas pelas cartas transcritas - voltadas para fortalecimento da imagem brasileira no contexto sul-centro e norte-americano destaca-se, no mesmo ano de 1927, a criação de uma Revista Brazil.

Tantas iniciativas, projetos e políticas, no entanto, não afloram nem ao livro América , nem tampouco à farta correspondência ${ }^{9}$ que, de Nova York, ele mantém com amigos. O silêncio do escritor sobre tais assuntos é completo.

Mais intrigante, porém, do que o silêncio de Monteiro Lobato sobre a pauta política de fortalecimento da imagem do Brasil, é seu silêncio sobre intercâmbios culturais entre a América do Sul e a do Norte que pesquisas contemporâneas sugerem estar em construção naquela época, delineando um promissor diálogo entre as Américas.

Neste diálogo avulta o papel desempenhado por Isaac Goldberg, figura muito presente nas relações literárias entre não apenas Brasil e

8. MUSEU DO ITAMARATY. SECRETARIA DE ESTADO DAS RELAÇÕES EXTERIORES Em 22 de Novembro de 1927 No PA/ 27 CONFIDENCIAL Índice: Movimento anti-americano na Conferencia da Havana.

9. Em Em busca do Lobato das cartas : a construção de ... (Tin, Emerson. Tese de Doutorado. IEL. Unicamp. 2007) Dr. Tin faz uma competente análise da correspondência lobatiana. Disponível em http://www.bibliotecadigital.unicamp.br/document/?code= vtlsoo0418763\&opt $=4$ 
Estados Unidos, mas entre a literatura latino-americana e os Estados Unidos. ${ }^{10}$

\section{Quem foi Isaac Goldberg?}

Foi um intelectual de alto quilate.

Nascido em 1887, faleceu em 1938. Estudou na Universidade de Harvard, onde bacharelou-se, fez seu Mestrado e seu Doutorado. Jornalista do Boston Evening Transcript era poliglota, incluindo-se Português e Espanhol entre os idiomas que dominava. Publicou inúmeros livros, ensaios e artigos sobre literatura latino-americana.

Para uma apresentação mais autorizada de Isaac Goldberg, com a palavra um brasileiro que o conheceu pessoalmente e que parece ter tido percepção acurada de seu papel como mediador cultural: Gilberto Freyre, intelectual brasileiro que nos anos vinte do século passado estudou Antropologia na Columbia University.

Numa das entradas de seu diário ${ }^{11}$, relativa ao ano de 1921, ele registra:

Venho a Boston a convite de Amy Lowell. Mas não deixo de visitar Goldberg. Almoço com o casal Goldberg. Almoço - diz-me ele de início - à moda dos judeus.

Dão-me de comer uma carne ( já não me lembra seu nome em yiddish) que me recorda o cozido brasileiro. Até onde irá - penso durante o almoço com os Goldberg - a influência do judeu sobre a comida brasileira? A "feijoada dormida "o "munguzá dormido", o quitute que se come depois de uma noite como que de repouso encoberto da iguaria, talvez seja reminiscência brasileira dos dias de quitutes encobertos e até secretos dos cristãos-novos.

Goldberg é homem de Harvard. Bacharelou-se em Harvard, seria ótimo professor de literaturas neo-hispânicas - inclusive a brasileira, na mesma Harvard. Por que não o querem na velha universidade onde outrora Longfellow ensinou literatura portuguesa, ao lado da italiana e da espanhola? Porque - explicou-me um anglo saxão - sua "personalidade judaica"é considerada

10. Algumas das reflexões aqui apresentadas relativamente às relações latinoamericanas de Monteiro Lobato retomam tópicos do Doutorado de Tahis de Mattos Albieri. São Paulo-Buenos Aires: a trajetória de Monteiro Lobato na Argentina [Tese de Doutorado. Universidade Estadual de Campinas. Instituto de estudos da Linguagem. 2007]. Disponível em http://cutter.unicamp.br/document/?code=000772097 Outras - relativas às relações norte-americanas de Monteiro Lobato retomam tópicos do Doutorado de Rosemary Paula Leite Carter , Monteiro Lobato acontece na América. Análise de duas transposições do conto $O$ engraçado arrependido " [tese de Doutorado. Universidade Presbiteriana Mackenzie, 2012] Cf. (Carter). Disponível em http:// mx.mackenzie.com.br/tede/tde_busca/arquivo.php?codArquivo=2533.

11. Gilberto Freyre Tempo morto e outros tempos [trechos de um diário de adolescência e primeira mocidade. 1915-1930]. São Paulo: Global. 206. p. 105-106. Agradeço ao Prof. Dr. Antonio Dimas as primeiras indicações relativas às relações entre Gilberto Freyre e Isaac Goldberg. 
“desagradável” pelos anglo-saxões. Em outras palavras, porque há em Harvard preconceitos anti-semitas.

Deficiência de que está livre Colúmbia que é, ao mesmo tempo, muito inglesa e muito anglicana - de acordo com sua tradição de escola fundada ainda por monarca inglês sob a proteção da coroa: ainda hoje o seu símbolo ou a sua insígnia - e amplamente cosmopolita. Inclusive com muita presença judaica. Pena que ao seu catedrático de Literatura Espanhola - Don Frederico de Onis, erudito espanhol dos mais completos na sua especialidade - falte o conhecimento de língua portuguesa e das literaturas de Portugal e do Brasil. Exatamente o conhecimento que tornaria Goldberg mestre ideal de literaturas hispânicas em qualquer grande universidade deste país ou da Europa.

O depoimento de Gilberto Freyre não apenas franqueia a seus leitores o pensamento complexo e instigante de um intelectual que, ainda bastante jovem, já elaborava uma teoria da miscigenação da cultura brasileira, mas revela ainda uma aguda e precoce compreensão do papel dos Estados Unidos - também América- nesta construção de uma identidade literária e cultural latino-americana.

Interessam-nos aqui alguns dos trabalho de Goldberg sobre literatura brasileira (que foram muitos), um dos quais levou, em 1922, o Monteiro Lobato escritor para o país no qual cinco anos depois, o Monteiro Lobato de carne e osso integraria o corpo diplomático da representação brasileira.

E é o silêncio de Monteiro Lobato relativo a esta sua precoce presença em letra de forma no mercado norte-americano que surpreende.

É a imagem literária de uma América Latina que, em 1921, parece já estar sendo delineada em Massachussets (em Roxbury), pelas mãos de Isaac Goldberg. Em 1921, ano da visita de Gilberto Freyre, Goldberg já estava bastante familiarizado com a literatura brasileira; é neste ano que ele publica pela International Pocket Library o volume Brazilian tales ${ }^{12}$.

Brazilian tales é obra extremamente interessante.

Na sequência de uma arguta apresentação da literatura brasileira, Goldberg monta uma antologia de contos informando, no entanto, seus leitores, que some of the tales first appeared, in English, in the Boston Evening Transcript and the Stratford Journal Boston. A informação instiga pesquisas que confirmem e localizem a publicação anterior dos contos.

12. Disponível em E: $\backslash$ 2010-2 $\backslash$ NOVEMBRO $\backslash$ Brazilian Tales, Translated by Isaac Goldberg.mht (projeto Gutenberg). Site consultado em 05.11.2010. 
O quadro abaixo, dos autores e textos presentes na antologia, indica, em rodapé e quando possível, sua publicação original em Português:

\begin{tabular}{|c|c|}
\hline AUTOR & TÍTULO EM INGLÊS \\
\hline Machado de Assis & ${ }^{13}$ \\
\cline { 2 - 2 } Medeiros $_{\mathrm{e}}$ & The Attendant's Confession \\
\hline $\begin{array}{c}\text { Mlbuque Teller Life } \\
\text { Albuerque }^{14}\end{array}$ & The Vengeance of Felix \\
\hline Coelho Neto $^{15}$ & The Pigeons \\
\hline Carmen Dolores $^{16}$ & Aunt Zezé's Tears \\
\hline
\end{tabular}

Monteiro Lobato não comparece a este livro cuja marca de originalidade é a presença de Carmem Dolores em época em que era rara, na tradição brasileira dos estudos literários, a menção à escrita feminina.

É no ano seguinte (1922), que Monteiro Lobato ganha espaço nobre na obra de Isaac Goldberg, um brazilianist avant la lettre. Em 1922 Goldberg publica pela Alfred A.Knopf o livro Brazilian Literature.

Com foreword de J.D.M. Ford - Professor de Harvard sob cuja orientação estudou Isaac Goldberg entre 1910-1912 -, o livro é dedicado a Burton Kline, que teria printed in the Boston Evening Transcript my first articles upon Spanish and Portuguese American letters. Prefácio e dedicatória trazem, assim, para cena mais personagens envolvidos nesta precoce incursão das letras brasileiras em território norte-americano. E ampliam o âmbito da tão necessária e urgente pesquisa da pré-história

13. Machado de Assis nasceu em 1839 e morreu em 1908. Ambos os contos foram publicados em Várias histórias, obra de 1896.

14. Medeiros e Albuquerque (1867-1934) escreveu os seguintes livros de contos: Um homem prático ( 1898), Mãe tapuia (1900), Contos escolhidos (1907), O assassinato do general. Até agora não foi possível identificar o original traduzido por Goldberg.

15. Coelho Neto (1864 - 1934) Até agora não foi possível identificar o original traduzido por Goldberg. http://www.cchla.ufpb.br/jornaisefolhetins/estudos/a literatura_nos_jornais_paraibanos.pdf informa que um conto de Coelho Neto intitulado Os pombos foi publicado em 20.01.1895 no jornal "o estado da paraíba"

16. CARMEN Dolores é pseudônimo de Emilia Moncorvo Bandeira de Melo (1852 1910). Isaac Goldberg informa que the story in this volume is taken from a collection entitled "The complex soul” http://www.releituras.com/cdolores_menu.asp registra 1934 como a data de publicação de Almas complexas 
dos Brazilianistas. Quem foram e o que publicaram J.D.M.Ford \& Burton Kline?

No foreword que escreve para o livro, o Professor Ford estabelece sugestiva articulação entre a publicação do livro e o centenário da Independência do Brasil. Este seu movimento aproxima - de um ponto de vista distinto do ponto de vista da correspondência diplomática anteriormente comentada, mas no mesmo sentido dela - o horizonte político de relações literárias entre países:

(...) to beautiful Rio de Janeiro many nations are sending their envoys with felicitations and gifts. Our own country, the United States of North America, is mindful of her duty and her privilege on this occasion, and accredited delegates are bearing her congratulations to her ever-faithful associate in the promoting of peace and fraternity throughout the Western Hemisphere ${ }^{17}$

Como se vê, aquela parceria Brasil/Estados Unidos que em 1927 vai expressar- se em correspondência diplomática como a transcrita, tinha antecedentes. O Brasil era um ever-faithful associate dos norteamericanos in the promoting of peace and fraternity throughout the Western Hemisphere, em anos vizinhos do término da 1a. Guerra.

Ao final de seu foreword, o texto do Prof. Ford sublinha a relevância das relações políticas e comerciais entre Brasil e Estados Unidos, articulando a elas a importância do intercâmbio literário e reforçando a aliança entre os países, papel que hoje talvez se traduza na força e na contemporânea planetarização da área acadêmica da Literatura Comparada e dos Estudos Culturais.

To his fellow-citizens of the United States of North America Dr. Goldberg now presents an opportunity of viewing aspects of the soul of a noble Southern land, their constant ally. Brazil's political and commercial importance they know well (...)

É ainda no mesmo texto que se insinua a feição americanista - no sentido mais amplo da expressão - que questões literárias parecem ir assumindo naquela época, talvez ainda assombrada pelos fantasmas da guerra de 1914-1918. Ao comentar o balanço positivo que Goldberg faz da literatura brasileira, o prof. Ford parece insinuar um certo sentimento anti-europeu, ingrediente que poderia cimentar relações globalmente americanas:

His laudation, moreover, is uttered without any tinge of that condescension which European critics deem it incumbent upon them to manifest when they pass judgment on the culture of North or South America (...) 
As mesmas inter-relações entre economia e cultura, política e literatura reaparecem no Preface de Goldberg, numa formulação que hoje - quase um século depois - continua de extrema atualidade:

Commercial opportunities, more than cultural curiosity, served to impart an impetus to the study of Spanish and soon we were reading fiction not only from Spain but from Spanish America. In so far as the mercantile spirit was responsible for this broader literary interest, it performed an undoubted service to art by widening our horizons, but one should be wary about overestimating the permanent gain.

Num estilo que tem suas passagens bem humoradas, Goldberg manifesta extrema familiaridade com autores e críticos brasileiros contemporâneos seus. Em diferentes momentos do texto, Monteiro Lobato é mencionado e estas menções recobrem as principais facetas lobatianas -além da de contista muito popular-manifestadas até 1922: proprietário e editor da Revista do Brasil, editor proprietário de uma editora comercial, e - de forma absolutamente surpreendente, para a novidade que representavam em 1922 as obras infantis lobatianas escritor para crianças.

Relativamente a este último gênero - que com impressionante argúcia Goldberg vê como porta de profissionalização para escritores - o livro de Goldberg não deixa de manifestar o preconceito com que até hoje livros para crianças e jovens são vistos em certos recantos da cidade das letras:

(...) we see how frequently even the major creative spirits of the country turn to the writing of text-books. Yesterday Olavo Bilac, fellow Parnassian of Francisca Julia, spared time for the labour; today Coelho Netto, Oliveira Lima, Monteiro Lobato do so. Again and again is one reminded what a sacrifice, what a luxury, is the creative life in a land that lacks anything like the creative audience (...)

Ao mesmo tempo, porém, em que desqualifica literatura infantil e livros escolares, Goldberg registra a irresistível atração que a escrita lobatiana para crianças desperta em maiores e menores de idade:

He is also the writer of several books for children. The best known of these is Narizinho Arrebitado or, as who should say Little Snuh-Nose, and with an appropriate blush I confess that the little girl's adventures among the flowers and creatures of her native land were responsible for the theft of some hours from the study of fatter, less childish, tomes (...)

Na análise do papel desempenhado por Monteiro Lobato na cultura brasileira, Goldberg registra, inicialmente, o extremo nacionalismo do escritor paulista. 
First and foremost he is the champion of the national personality. And by that same token he becomes the enemy of undue foreign influence upon the nation. As one reads his numerous short stories, his crisp and vigorous criticisms and his essays, one comes to the realization that, as far as Lobato is concerned, foreign influence is chiefly French and in large measure to be condemned (...)

O crítico não deixa de assinalar, no entanto, o frequente uso que Lobato faz de epígrafes estrangeiras (inclusive francesas) em sua obra. Ao frisar este ponto, que pode ser considerado uma contradição lobatiana , Goldberg abre uma porta que conduz a leituras bastante produtivas da obra do escritor paulista. Ao autor de Urupês ajusta-se como luva, a auto-apresentação vulcânica do eu-lírico (marioandradino) de Remate de males: Sou trezentos, sou trezentos e cinqüenta.

Monteiro Lobato foi, realmente, múltiplo.

Goldberg parece ter se fascinado por esta figura múltipla e contraditória, a ponto de três anos depois - em 1925 - publicar uma antologia de seus contos, um dos quais já havia merecido seus aplausos no livro de 1922:

Tales like A Modern Torture (in which a rural dabbler in politics, weary of his postal delivery "job," turns traitor to the old party and helps elect the new, only to be "rewarded" with the same old "job") are rare in any tongue and would not be out of place in a collection by Chekhov or Twain.

$\mathrm{Na}$ menção ao russo Chekhov e ao norte-americano Twain como padrões de excelência literária aos quais Goldberg compara (positivamente) Monteiro Lobato, talvez se possa ler a ruptura com a hegemonia européia como padrão de excelência, à qual referia-se Ford.

Mesmo as restrições que em 1922 Goldberg faz ao escritor paulista são fecundas e inspiradoras - quase um século depois- para releituras lobatianas. Ao apontar o volume e a regularidade da produção lobatiana, Goldberg assinala a falta, nela, de um certo artesanato da escrita literária.

(...) It may be gathered that Lobato writes -or rather reprints- too much; for plenty of good journalism should be left where it first appeared and not be sent forth between covers. Also, in an appreciable amount of his work, his execution lags behind his intention, owing in no small measure to a lack of self-discipline and an artistically unripe sincerity (...)

17. http://www.questia.com/read/1387293?title=Brazilian\%2oLiterature\%20 (1922) .Deste site são extraídas todos os textos aqui transcritos. Site consultado em 05.11.2010 
Este artesanato efetivamente ausente do projeto literário de Lobato - tal como este projeto se dava a conhecer (e estava in progress) em 1922 $\mathbf{2}^{18}$, encontra respaldo no discurso do século XXI sobre literatura?

Mais adiante, ilustrando a tese da escrita militante de Monteiro Lobato, Goldberg traduz um longo trecho de Ideias de Jeca Tatu, obra de 1919 que pode talvez ser considerada a mais sistemática exposição das ideias estéticas de Monteiro Lobato. Já quase ao final do capítulo, Goldberg comenta carta que teria recebido do escritor e da qual constaria uma espécie de mini-biografia, o que sinaliza que já havia relações pessoais entre o norte americano e o brasileiro anteriores a 1922.

Estas relações são, até agora pouco conhecidas, bem como pouco conhecidas - embora sinalizadas por Albieri- são também as articulações que Goldberg diz reconhecer entre a obra lobatiana e procedimentos que ele acredita encontrar no que chama de um grupo de Buenos Aires:

For of pure literature there is little in the young Saint-Paulist. I fear that, together with a similar group In Buenos Aires, he underestimates the esthetic element in art, confusing It, perhaps, with the snobbish, aloof, vapoury spirits who have a habit of infesting all movements with their neurotic lucubrations (...) .

Na antologia lobatiana de 1925, ele nomeia alguns membros deste grupo dando, portanto, concretude à postulada relação triangular entre Brasil, Argentina e Estados Unidos.

Esta aproximação que Goldberg estabelece entre a literatura do Brasil e a da Argentina torna promissoras pesquisas que esteiem na materialidade de dados e de documentos esta precoce internacionalização da literatura brasileira e este igualmente precoce ingresso da América Latina nos estudos literários norte-americanos. Esta até agora pouco conhecida rede de relações torna-se ainda mais sugestiva por Goldberg ser menção constante na correspondência entre Monteiro Lobato e escritores argentinos, particularmente na correspondência do escritor paulista com Manuel Galvez e Horacio Quiroga.

Com este último, inclusive, Lobato parece ter discutido caminhos possíveis - dos quais, aparentemente, Isaac Goldberg não participa - para lançamento de seus (= dele e de Quiroga) livros nos Estados Unidos:

18. Em Monteiro Lobato livro a livro: obra infantil (São Paulo: EDUNESP-IMESP, 2008 [Prêmio Jabuti $20091^{\circ}$. Lugar Teoria/Crítica Literária. Livro do ano não ficção]) a discussão de diferentes versões das obras infantis lobatianas talvez relativize esta hipótese ao apontar e discutir as inúmeras variantes de sua obra infantil. A pesquisa sugere que ao longo da vida o escritor desenvolve e aprimora um projeto de escrita literária. 
Buenos Aires, octubre 13, $1922{ }^{19}$

Querido Lobato:

Cuando me disponía a escribirle hoy, llega su carta. Sabrá que llegué aquí anteayer, pues quedé en Montevideo 8 o 10 días. Estoy por fin descansando, después del horrible traqueteo diplomático de feliz memoria.

Buen recuerdo la foto pauliceana. No falta por aquí algún mal intencionado que asegura que he ido a San Pablo a surtirme de nuevos cuentos, que luego firmo como míos. Con la postal adjunta se convencerán más aún.

Antes que me olvide: dígame si no se opone a que traduzca y publique donde me sea posible EL PADRE DE LA GUERRA. Para mayor coincidencia de nuestras tristes personas, también escribí yo un artículo sobre el mismo asunto, que le remito con ésta. No pude meter dicho artículo en LA NACION, por razones que comprenderá Ud. de sobra. Don Leopoldo Lugones se había desatado en una vergonzosa campaña ultra francesa en aquel diario, y de rabia le contesté sin nombrarlo. Otra vez le he de manda a Ud. otros artículos sobre el mismo tema. Contésteme en seguida a este respecto. Yo le pondría una especie de prologuillo.

Le mando también la traducción del contrato que hice por CUENTOS DE LA SELVA y una de las últimas cartas de N. York recebidas, donde se enterará de las esperanzas que - Ud. y yo por lo menos - debemos tener de aquel mercado. Devuélvame todos los papeluchos. Mañana escribiré a la THE FOREIGN PRESS SERVICE hablándole de Ud. con el respeto debido. Y si luego Ud. hace traducir y revisar allí mismo uno de sus cuentos más yankizables, les facilitaría el trabajo a aquellas gentes, cuando se pongan en pour parler.

(...). Quiroga

Alguns meses depois, nova carta de Quiroga retoma o tema e deixa transparecer a perspectiva pragmática pela qual ele e Monteiro Lobato viam o mercado norte-americano. A leitura cruzada de Goldberg com latino-americanos parece abrir trilhas para um conhecimento mais nuançado dos imaginários recíprocos do norte e do sul de nuestra América:

B. Aires, noviembre 14 de $1922^{20}$

Mi querido Lobato:

Recibida carta. Junto con la suya me llegaba la traducción de CUENTOS DE LA SELVA, que le envie. Trabaja con gran gusto aquella gente. Han hecho lo que ellos llaman "adaptación libre", y en verdad se trata de eso. Pero a lo que entiendo han conservado bastante bien el espíritu del libro. El traductor no me lo oculta, tampoco. Cuando lleguen noticias editoriales, se las comunicaré. Parece también que en E.U. hay tendencia a hacer libros para chicos en formato para grandes. Tal, el libro mío.

Muy bien por la HOUSTON PUBLISHING. Aprovéchese de ella, pues yo no puedo, por ser la FOREING PRESS mi agentes exclusivos en E.U. Y todavía nada

19. Cf. (CEDAE MLb 3.2.00292 cx6)

20. Cf. (CEDAE MLb 3.2.00297 cx6) 
he podido hacer en cuentos, pues me comunican que a pesar de haber tratado la colocación do A LA DERIVA en un magazine de allá, a última hora hallan inconvenientes los del magazine, por la resistencia cada vez mayor de aquel país a aceptar cosas extranjeras de ambiente. ¡Estamos frescos, amigos! ¿Querrán que les demos estúpidas historietas de amor, como las que hacen ellos? En fin, tantos Ud, y puede ser que por el HOUSTON PUBLISHING se averigue algo de bueno. El pago me parece magnífico. Eses 25 pesos oro suponen 75 u 80 nuestros, lo que es enorme tratándose de una traducción... que podrían muy bien robar. Tal como yo lo tendré al corriente de lo que pase por mi sector, infórmeme Ud del suyo. Al llegar escribía a la FOREIGN PRESS a su repecto, pero aún no he tenido respuesta. No nos apuremos, amigo.

(...) Quiroga

Seguindo o conselho de Quiroga a seu colega brasileiro, no nos apuremos.

Ao invés disso, retornemos ao ano de 1921. Em junho deste ano, em carta a um amigo enumerando as traduções do livro Urupês, Monteiro lobato informa que Nos Estados Unidos quer traduzi-lo Isaac Goldberg, informação repetida em novembro do mesmo ano a Galvez ${ }^{21}$ também comentando que Goldberg publicou um artigo sobre mim num jornal americano. Excelente propagandista e ótimo crítico.

Estas amostras de relações fraternas de Monteiro Lobato com Isaac Goldberg afloram na correspondência pessoal do escritor brasileiro e ganham registro bem mais formal quando - deixando a esfera privada da correspondência pessoal - afloram à esfera pública: a Revista do Brasil.

Esta revista, cujo proprietário e editor era - recordemos - Monteiro Lobato, em edição de 1921 (vol. XVIII, ano VI, n. 72) publica um longo artigo que apresenta Isaac Goldberg como notável crítico norte-americano, autor de uma obra recente "Studies in Spanish-American literature" hoje especializado em estudos sul-americanos, transcrevendo a seguir o que informa ser um artigo que Goldberg publicou no Evening Boston sobre Monteiro Lobato.

O artigo em português, que ocupa várias páginas da revista parece coincidir com longos trechos do estudo sobre Lobato que, em 1922 -como já mencionado- Goldberg incluirá em Brazilian Literature e que depois também aproveitará na apresentação dos três contos de Lobato que publica em Brazilian short stories de 1925.

Vale a pena um cotejo entre todas estas versões que aponte e discuta o que sai, o que fica e o que se altera em cada uma delas .

\footnotetext{
21. Conferir Albieri. Op. Cit. Disponível em http://cutter.unicamp.br/ document/?code $=000772097$
} 
Na proposta deste cotejo, retorna-se ao eixo de partida deste ensaio: por que - ao menos até onde as pesquisas chegaram hoje - Isaac Goldberg não faz parte do horizonte norte-americano que Monteiro Lobato viveu entre 1927 e 1931? Por que a obra Brazilian short stories, que teria feito Monteiro Lobato circular na América antes de sua chegada a Nova Iorque não é jamais mencionada pelo escritor? Como conciliar o Monteiro Lobato, funcionário do governo brasileiro em New York, que tagarela irreverentemente sobre tantos assuntos com o silencioso Monteiro Lobato cidadão da cidade das letras, já em circulação, em Inglês, nos Estados Unidos?

Retomando o que até aqui se viu, é ainda em 1921 que - através de artigo de Goldberg na imprensa de Boston-começam a tomar corpo os fios que podem tecer a realização do sonho literário norte-americano de Monteiro Lobato.

Nessa época, ele era o poderoso editor-proprietário da prestigiosa Revista do Brasil que republica o artigo em que Goldberg - de Boston - apresenta sua obra para o público norte-americano, antecipando o extenso estudo de sua obra que publica em 1922 e o lançamento em setembro de 1925, como número 733 da coleção Little blue books (editado por Haldeman Julius ${ }^{22}$ ) de Brazilian short stories, livro que se compõe de três contos de Monteiro Lobato: Modern torture, The penitent wag e The plantation buyer, cujo prefácio, datado de 1924 indica Roxbury (Mass) como berço do texto.

Estes contos que integram a antologia vêm de Urupês ${ }^{23}$, obra de estreia de Monteiro Lobato no Brasil, publicada em 1918. Cada um deles

22. A coleção é definida como uma "series of (...) small 31/2 x 5 inch paper covered booklets published in Girard ( Kansas) [...] printed in standardized lengths of 32, 64, 96 and 128 pages, (...) the Little Blue Books were explicity conceived as agency of popular culture" Cf. Herder, Dale M. Haldeman-Julius, The little Blue Books, and the Theory of Popular Culture. I Journal of Popular Culture. Vol. IV, Spring 1971, n 4 p. 881- 891. Para uma discussão mais aprofundada da coleção cf. Rosemary Paula Leite Carter, Monteiro Lobato acontece na América. Análise de duas transposições do conto O engraçado arrependido" [tese de Doutorado. Universidade Presbiteriana Mackenzie, 2012] Cf. (Carter). Disponível em http://mx.mackenzie.com.br/tede/tde_busca/arquivo.php?codArquivo=2533.

23. Os três contos fazem parte primeira edição de Urupês. Modern torture é a tradução de Um suplício Moderno; The penitent wag é a tradução de $O$ engraçado arrependido e The plantation buyer a tradução de $O$ comprador de fazendas. $O$ engraçado arrependido e $O$ comprador de fazendas tiveram publicação anterior à sua inclusão em Urupês. Com o título A gargalhada do coletor, o primeiro foi publicado em abril de 1917 na Revista do Brasil e mantendo o mesmo título com que aparece no livro, o segundo foi publicado em março de 1918 no mesmo periódico. 
foi traduzido para Inglês por uma até agora não identificada woman friend of Lobato resident in Brazil.

E mais não se sabe da história deste livro.

O que Monteiro Lobato achou da publicação? Porque estes e não outros contos? Na obra de Goldberg de 1922, o conto O Jardineiro Timóteo parece gozar da estima do organizador da antologia, porém não faz parte dela. Não faz parte dela por razões talvez próximas das que obstaram a tradução para o inglês do romance $O$ presidente negro, a propósito do qual em 1927, Goldberg retorna à correspondência lobatiana:

Meu romance não encontra editor. (..) Os originais estão com o Isaac Goldberg, para ver se há arranjo. Adeus, Tupy Company! (05.09.1927) 24

A perspectiva pela qual em $O$ presidente negro Monteiro Lobato tematiza a questão racial na sociedade norte-americana, inscrevendo o enredo no então remoto ano de 2228 é considerada inadmissível por um editor que aconselha Monteiro Lobato a enveredar por outros caminhos se quiser ser editado nos Estados Unidos:

Em papel timbrado da Palmer Literary Agency (6362 Hollywood Boulevard, Hollywood Califórnia), em 17 de novembro de 1927, William David Ball, editor in Chief escreve a Monteiro Lobato:

\author{
Mr. J.Manturo (sic) Lobato ${ }^{25}$ \\ $205-24$ th St \\ Jackson Heights, LI, NY \\ Dear Mr. Lobato:
}

(1) It has been with more than an average degree of interest that I have you're your story THE CLASH OF THE RACES for the material has been entertainingly presented and indicates a rich creative imagination. Unfortunately, however, the central theme is based on subject matter that is particularly difficult to present in this country for it is likely to awaken the bitterest kind of partisanship, and for that reason, publishers are invariably loathe to present it to the reading public.

Relativamente ás variantes dos contos lobatianos, consultar Martins, Milena Ribeiro Lobato edita Lobato : história das edições dos contos lobatianos. Doutorado. Universidade Estadual de Campinas. Instituto de Estudos da Linguagem. 2003 www.biblioteca digital. unicamp.br//document/?code=vlisooozo//66 e também Quem conta um conto ... aumenta, diminui, modifica. O processo de escrita do conto lobatiano. Mestrado. Universidade Estadual de Campinas. Instituto de Estudos da Linguagem. 1998 www. unicamp.br/iel/memoria/projetos/tese 13.html

24. MONTEIRO LOBATO A barca de Gleyre (Correspondência de Monteiro Lobato com Godofredo Rangel). SP: Editora Brasiliense. p. 304

25. 0309_cV1_02, 0309_cv1_03, 04, 0309_CV1_05 
Your preliminary chapters are very well handled and pave the way for the amazing revelations of Prof. Benson and his daughter in a convincing fashion. You have also succeeded very well in maintaining a thread of intimate personal interest through the love of the narrator Ayrton Lobo for Jane Benson, which would prevent the reader from losing himself in the mazes of fantastic events (2) Had the main complications of your story dealt with almost anything else rather than the negro question, there would have been a better opportunity of placing the manuscript with a publisher. Had you allowed your imagination to play with some mythical invasion or had events dealt particularly with the struggle between the sexes, leading to some significant and entertaining solution instead of being subsidiary action as is presented here, there might be a possibility of finding a market depending of course on the novelty of the solution and the skill in treatment. But when it comes to the negro question, and the suggestion that they are to be entirely exterminated, not even the fact that this event is 300 years in the future, would soften it in the minds of negro readers. The poignancy of the situation and the appeal with which you have endowed the chief negro character James Ridden (sic), would only serve to heighten the undesirable factors in the case. Where you dealing with the invasion of an alien nation, or race,

(3) the reaction would be quite different; but the negro is an American citizen, an integral part of national life, and to suggest his complete extermination through the superior wit and skill of the white race would lead to almost as violent dissention in the mind of readers, as would the presentation of a conflict between two political parties or two religioous denominations in which one extirpated the other.

There is no doubt of your ability. Your work reveals a keen sense of the dramatic and highly imaginative qualities. As you proceed with your study of English and of creative writing, I should advise you to pay particular attention to the type of material that is used extensively by American publishers. Canvas the market thoroughly, for this will assist you greatly in your choice of subject material, as you proceed with your own work

(4) Do not look upon this manuscript as lost effort by any means. Keep it on file and later on, when you have been able to gain a fresh perspective on the idea, it is possible that you may be able to retrieve much of the story material, interweaving it with some other problem or thematic idea.

I shall look forward with much interest to seeing other examples of your work, and I hope that you are going to derive a vast amount of pleasure and benefit from your study of the course when you are able to proceed with it.

With kindest wishes, I remain

Sincerely,

William David Ball.

Editor in Chief

É, pois, neste contexto de uma recusa editorial da publicação norteamericana de $O$ presidente negro, que Monteiro Lobato parece apostar suas últimas fichas em Isaac Goldberg, como registra a carta de 05 de setembro. 
Mas Isaac Goldberg - antes de ser a última esperança de Lobato para o acalentado projeto de publicar o romance na América - foi o primeiro a publicar seus contos no mercado norte-americano. O que torna mais intrigante ainda a questão: por que Monteiro Lobato não comenta a edição norte-americana de sus contos? Como nasceu e como circulou o modesto livro das Brazilian short stories? Quantos exemplares vendeu? Quem era a woman friend of Lobato que traduziu os contos? O exemplar em poder da família - bastante deteriorado - tem correções manuscritas que lembram a caligrafia de Lobato.

\section{Serão dele?}

São perguntas cujas respostas com certeza, iluminarão o papel de Isaac Goldberg na constituição deste precoce, precário e abortado projeto de uma rede literária que unisse as Américas, sonho até hoje generoso e - cada vez mais - oportuno.

\section{REFERÊNCIAS BIBLIOGRÁFICAS}

ALBIERI, Tahis de Mattos. São Paulo-Buenos Aires: a trajetória de Monteiro Lobato na Argentina. Tese de Doutorado. Universidade Estadual de Campinas. Instituto de estudos da Linguagem. 2007. Disponível em http://cutter.unicamp.br/ document/?code $=000772097$

AZEVEDO, Carmen; CAMARGOS, Márcia; SACHETTA, Vladimir. Monteiro Lobato: Furacão na Botucúndia. São Paulo: Senac. 1997.

BIGNOTTO, Cilza. Monteiro Lobato e a edição de Mistério. In Adami, Antonio; Heller, Bárbara; Cardoso, Haydê Dourado de Faria (org). Mídia, Cultura e Comunicação. 2. São Paulo: Arte \& Ciência. 2003. p. 303-311).

BIGNOTTO, C.C. Novas perspectivas sobre as práticas editoriais de Monteiro Lobato (1918-1925). Tese de Doutorado. Universidade Estadual de Campinas. Instituto de estudos da Linguagem. 2007. Disponível em http://cutter.unicamp.br/ document $/$ ?code=vtlsooo 410260

CARTER, Rosemary Paula Leite. Monteiro Lobato acontece na América. Análise de duas transposições do conto $O$ engraçado arrependido [tese de Doutorado. Universidade Presbiteriana Mackenzie, 2012] Cf. (Carter). Disponível em http://mx.mackenzie. com.br/tede/tde_busca/arquivo.php?codArquivo=2533.

CAVAlHEIRO, Edgard. Monteiro Lobato: Vida e obra. São Paulo: Editora Brasiliense, 1955 .

FREYRE, Gilberto. Tempo morto e outros tempos (trechos de um diário de adolescência e primeira mocidade. 1915-1930) São Paulo: Global. 
GOLDBERG, Isaac. Brazilian literature. NY Core Collection Books. 1922. Disponível em www.questia.com/read/1387293?title=Brazilian\%2oLiterature\%20(1922). Site consultado em 05.11.2010.

. Brazilian Tales. Disponível em E:\2010-2\NOVEMBRO \Brazilian Tales, Translated by Isaac Goldberg.mht Site consultado em 05.11.2010.

HERDER, Dale M. Haldeman-Julius, The little Bluie Books, and the Theory of Popular Culture. I Journal of Popular Culture. Vol. IV, Spring 1971, n 4 p. 881-891.

LAJOLO, M. A Brazilian writer (Monteiro Lobato) in New York apud Brazil/Brasil (o103751X) volume 34,série 19,2006. p.17-31.

. Monteiro Lobato: um brasileiro sob medida. São Paulo: Ed. Moderna. 2000.

. Um brasileiro em trânsito apud Ilha do Desterro (UFSC) 2009 p. 37-57. ISSN o1014846 .

LAJOLO, M. \& CECCANTINI, J.L. (orgs) Monteiro Lobato livro a livro: obra infantil. São Paulo: EDUNEST-IMESP, 2009.

LAJOLO, M. e ZILBERMAN, R. O preço da leitura. São Paulo: Ática, 2001.

ASSIS, Machado de. Obra completa. 3 vol. RJ Ed. José Aguilar LTDA. 1962 . vol I p. 56o. A primeira edição da obra é de 1880 .

MARTINS, Milena Ribeiro Lobato edita Lobato: história das edições dos contos lobatianos. Doutorado. Universidade Estadual de Campinas. Instituto de Estudos da Linguagem. 2003 www.biblioteca digital. unicamp.br//document/?code=vlisooo3o//66

MARTINS, Milena Ribeiro Quem conta um conto ... aumenta, diminui, modifica. O processo de escrita do conto lobatiano. Mestrado. Universidade Estadual de Campinas. Instituto de Estudos da Linguagem. 1998 www.unicamp.br/iel/memoria/projetos/ $\underline{\text { tese } 13 . \mathrm{html}}$

LOBATO, Monteiro. América. São Paulo: Editora Brasiliense Ltda.1956 [Obras completas de Monteiro Lobato. 1a. série Literatura geral. Vol. 9] (1a.ed:1931).

.Cartas escolhidas (prefácio de Edgard Cavalheiro). São Paulo: ed. Brasiliense 6a. ed. 1970 p.135 carta de 04.07.1928).

.Mr. Slang e o Brasil (Colóquios com o inglês da Tijuca). São Paulo: Editora Brasiliense. 1956 (1a. ed.1927). . Ideias de Jeca Tatu. São Paulo: Ed. Brasiliense. 1957.

. A barca de Gleyre. 20. Tomo. São Paulo: Editora Brasiliense, 1957.

NYT (1857-Current file) May 22, 1927. ProQuest Historical Newspapers The New York Times.p. E1)

TIN, Emerson Em busca do Lobato das cartas : a construção de ... (Tin, Emerson. Tese de Doutorado. IEL. Unicamp. 2007 ). Disponível em http://www.bibliotecadigital. unicamp.br/document/?code=vtlsooo $418763 \&$ opt $=4$ www.gutenberg.org/dirs/etextos/hnfrdı.txt NY Doubleday, Page \& Company. www.unicamp.br/iel/memoria www.unicamp.br/iel/monteirolobato www.wirednewyork.com/skyscrapers/whitehall/default.htm 